\title{
Inaccurate mimicry and predator ecology
}

Batesian mimicry, the resemblance of palatable organisms to an aversive model, is a well documented phenomenon in biology. Resemblance to the model, however, frequently seems to be inaccurate for unknown reasons (Edmunds, 2000). Recently, Johnstone (2002) modelled a scenario where kin selection can lead to the evolution of inaccurate mimics. The model assumes that if mimics are relatively frequent compared to models or if attacking the model incurs only moderate costs, the predator's incentive to attack will be high. As a consequence, the evolutionarily stable attack rate of a predator increases with an increasing local similarity of mimics to their model. Johnstone argued that under conditions where predation risk of the mimic increases with local similarity to the model, kin selection can favour the evolution of inaccurate mimics.

Here, we would like to suggest an addition to the model that may improve its resemblance to nature. In accordance with Lima (2002), we want to emphasize that expected outcomes of predator-prey interactions can radically change when predators are allowed to respond strategically to prey profitability. We suggest that a realistic estimate of predation risk would have to include not only attack rate but also predator density. Predator density is likely to be influenced by the net benefit of predation and will thus differ between patches with accurate and inaccurate mimics. In Johnstone's model, predators were assumed to optimize attack rate in individual patches, but patch choice or other predator responses to the available net benefit were not included. Generally, predators should prefer patches providing the largest net benefit. In the case of accurate and inaccurate mimics, the net benefit for predators to forage in patches with relatively inaccurate mimics will be higher because the risk of attacking models is reduced there, compared to patches with more accurate mimics. Thus, predators able to choose between patches that differ in local similarity between model and mimic should forage preferentially in areas with lower local similarity. Unspecialized predators may even refrain completely from attacking in patches with accurate mimics if increasing local similarity to the model leads to a lower net benefit in comparisons to the profitability of available alternative prey species. Under such conditions, an increased local frequency of predation resulting from an increased local net benefit to predators in patches with less complete mimicry thus at least has the potential to override the impact of kin selection proposed by Johnstone (2002).

Even if predators are incapable of habitat selection, predator reproduction and thus predator density is likely to depend on the net benefit predators can extract from their foraging. To estimate the effect of predator density on the evolutionarily stable level of dissimilarity $(d *)$ under such a scenario, we assumed an ideal free distribution of predators with respect to the available net benefit. This means that predator density is assumed to be directly proportional to the locally available net benefit and that predator density therefore increases with decreasing similarity between model and mimic. Exactly as described in Johnstone's model we calculated the evolutionarily stable level of dissimilarity by using $\bar{d}$, the local typical similarity between models and mimics. However, we used predation rate, the product of attack rate and predator density instead of the attack rate to calculate $d *$ (under the assumption $d *=\bar{d}=d$, see Johnstone (2002) for details). With an iterative process, we estimated $d *$ by calculating those values of $\bar{d}$ that satisfy the following equation:

$\frac{\partial A(d, \bar{d})}{\partial d}+r \frac{\partial A(d, \bar{d})}{\partial d}=0$.

Here, $r$ is a kin selection coefficient (see Johnstone (2002) for definition), and $A(d, \bar{d})$ denotes the product of predator density and attack rate, as described in the following equation:

$$
\begin{aligned}
A(d, \bar{d})= & {\left[p b\left(1-Z\left(-\frac{K}{\bar{d}}-\frac{\bar{d}}{2}\right)\right)\right.} \\
& \left.-(1-p) c\left(-\frac{K}{\bar{d}}+\frac{\bar{d}}{2}\right)\right]\left(1-Z\left(\frac{K}{\bar{d}}-\frac{\bar{d}}{2}-d\right)\right)
\end{aligned}
$$

(Here, the first factor denotes relative predator density, calculated from the locally available net benefitbenefits of attacking mimics minus costs of attacking models - and the second factor gives the attack rate as calculated by Johnstone (2002). In the above equation, $Z$ denotes the cumulative normal distribution function, see Johnstone (2002) for definition of this and the other variables).

As in the original model, we received evolutionarily stable imperfect mimicry $(d *>0)$ for all values of $K>0 \quad(K$ is the incentive of attack, defined as 


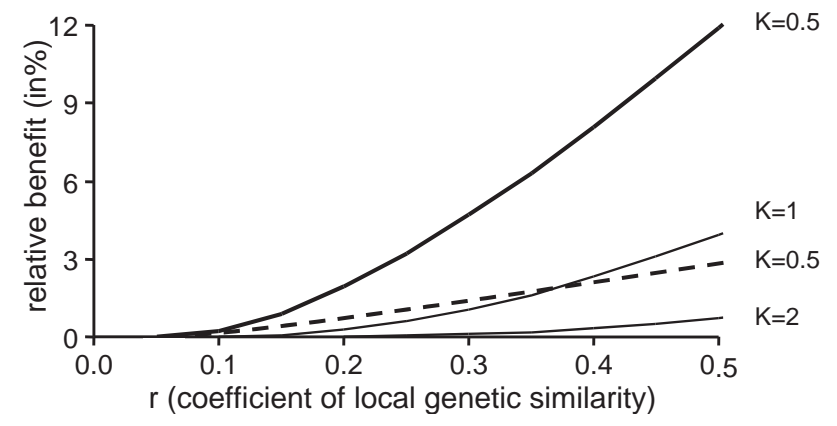

Fig. 1. Theoretical benefit of discrimination for predators (relative to indiscriminatory foraging) at the evolutionarly stable level of dissimilarity $d *$ between mimics and models as a function of the coefficient of local genetic similarity (all variables as defined by Johnstone, 2002). The three solid curves give the benefits expected for three different levels of $K$, the incentive of attack. The effect an inclusion of predator density into the estimation of the evolutionarily stable level of dissimilarity has on the potential benefit of prey distinction by predators, can be seen by comparing the hatched curve with the thick solid curve (note that $K=0.5$ for both thick curves).

$K=\ln [b * p /(c *(1-p))]$ where $b$ and $c$ are the benefits and costs of consuming mimics and models, and $p$ the relative frequency of mimics), but with reduced $d *$ compared to Johnstone's results. To show the effect of including predator density into the model, we estimated the benefit the distinction between models and mimics provides at the evolutionarily stable level of dissimilarity (relative to indiscriminate foraging) and compared it with the benefit according to Johnstone's original model. The inclusion of predator density in the model clearly decreased the potential benefit for the foragers to distinguish between models and mimics (Fig. 1). Any costs of distinction that exceed this reduced benefit, for example an increased handling time, should therefore lead to indiscriminative foraging. Under such conditions, mimicry has lost its function and selection might lead to camouflage, to the mimics extinction, or to mimicry of more dangerous models.

Summarizing the results of our reanalysis of Johnstone's model, we conclude that the potential of kin selection promoting the evolution of inaccurate mimics may be further limited to those cases where predator density is not influenced much by the locally available net benefit, where $r$, the coefficient of local genetic similarity from Johnstone's model is relatively large, and where the distinction between mimics and models only incurs minor costs.

\section{References}

Edmunds, M., 2000. Why are there good and poor mimics? Biol. J. Linnean Soc. 70, 459-466.

Johnstone, R.A., 2002. The evolution of inaccurate mimics. Nature $418,524-526$.

Lima, S.L., 2002. Putting predators back into behavioral predatorprey interactions. Trends Ecol. Evol. 17, 70-75.

K. Reinhold ${ }^{\mathrm{a}, \mathrm{b}}$
a Department of Zoology, Animal Behaviour,
University of Zürich, Winterthurerstr. 190,
CH-8057 Zürich, Switzerland

E-mail address: kreinhold@evolution.uni-bonn.de

L. Engquist ${ }^{\mathrm{b}}$

${ }^{\mathrm{b}}$ Institute of Evolutionary Biology \& Ecology, University of Bonn, An der Immenburg 1, 53121 Bonn, Germany 